\title{
Some Ideas about the Thermal Equilibrium in the Biosphere and the Entropy Variation Ascribed to Changes in the Radiations Wavelengths
}

\author{
Jaime González Velasco \\ Universidad Autónoma de Madrid, Facultad de Ciencias, Cantoblanco, Madrid, Spain \\ Email: jaime.gonzalez@uam.es
}

Received 17 August 2015; accepted 1 November 2015; published 4 November 2015

Copyright (C) 2015 by author and Scientific Research Publishing Inc.

This work is licensed under the Creative Commons Attribution International License (CC BY). http://creativecommons.org/licenses/by/4.0/

\section{(c) (i) Open Access}

\begin{abstract}
An explanation is given for the thermal equilibrium in the biosphere, which is based in the equality between the thermal energy received from the sun and the thermal energy reemitted from the atmosphere to the space. In order to understand the origin of the energy that gives rise to the processes and phenomena taking place in the biosphere, it is necessary to take into account the free energy represented by the product of temperature times the change in entropy, $T \Delta S$, whose magnitude can be attributed to the variation experimented by the wavelengths (or, consequently, the frequencies) of the radiations composing the radiation spectrum received from the sun compared with the radiation spectrum reemitted from the biosphere into the space. A simple discussion allows to predict that the entropy increase driving the processes is connected with a spontaneous conversion of high frequency radiations (with lower "content" of entropy) in radiations of lower frequencies (with higher "content" of entropy). A consequence of this is that high frequency radiations would correspond to more ordered states and, therefore, to less probable states than those corresponding to radiations of lower frequencies.
\end{abstract}

\section{Keywords}

Biosphere, Thermal Equilibrium, Entropy, Radiation Frequency and Entropy

\section{Introduction}

The discussion on the conditions that should be accomplished for the establishment of the thermal equilibrium in

How to cite this paper: Velasco, J.G. (2015) Some Ideas about the Thermal Equilibrium in the Biosphere and the Entropy Variation Ascribed to Changes in the Radiations Wavelengths. Open Journal of Physical Chemistry, 5, 106-109. 
the biosphere leads to an apparent paradox. The thermal equilibrium which allows an average temperature of $15^{\circ} \mathrm{C}$ in the biosphere [1] is based in the reemission to the space, in the form of radiating energy, with a power spectrum corresponding, approximately, to the emission spectrum of a blackbody at a temperature of $15^{\circ} \mathrm{C}$, of the same amount of thermal energy that it is absorbed from the sun by the terrestrial atmosphere. This is the process, according to which the thermal equilibrium in the biosphere is admitted to take place. Nonetheless, before the radiation is reemitted, a large number of processes and phenomena take place in the biosphere, whose development requires the degradation of the energy to another, lower degree. The lowering of degree in the energy is a measure of the amount of free energy that is necessary to give rise to all the processes required to maintain the functioning of the biosphere, and this free energy is represented by the term $\mathrm{T} \Delta \mathrm{S}$, i.e., by the variation in entropy and temperature which experiments the incident solar radiation with respect to the spectrum of radiations reemitted to the space surrounding the Earth.

The electromagnetic radiation reaching the outskirts of the terrestrial atmosphere presents a distribution of power by surface and frequency unit which is very similar to the radiation spectrum corresponding to a black body at $6000 \mathrm{~K}$ and arrives at a rhythm of around $1360 \mathrm{~W} \cdot \mathrm{m}^{-2}$. On the other hand, in order that the temperature in the biosphere remains constant along the time, the energy should be irradiated from it to the surrounding space at the same rhythm at which it is absorbed, i.e., it is necessary to reach a thermal equilibrium. Since the average temperature of the biosphere is estimated to be $15^{\circ} \mathrm{C}$, the spectrum of the energy irradiated is similar to the corresponding to a black body at a temperature of $15^{\circ} \mathrm{C}$, which is characterized by a distribution of radiation of much lower frequencies (frequencies located in the range of the far infrared) than the spectrum of the electromagnetic radiation coming from the Sun, whose frequencies are located in the ultraviolet and visible ranges. Thus, the phenomena occurring in the biosphere must be the consequence of the lowering of the frequency which takes place in the electromagnetic radiation and the change in entropy responsible for the degradation of the electromagnetic energy should be attributed to the change in the frequency experimented by the different radiations composing the solar spectrum reaching the Earth, in comparison with the lower frequencies (or larger wavelengths) corresponding to the radiation spectrum emitted by a black body at $15^{\circ} \mathrm{C}$.

\section{Thermodynamic Interpretation of the Transformations Taking Place in the Biosphere}

In order to explain how a lowering of the frequency of a radiation is able to give rise to the multiples transformations taking place in the biosphere it is necessary to resort to the Thermodynamics. According to the arguments exposed in the former paragraph, at a high frequency radiation should correspond a low value of the entropy, whereas at a radiation of low frequency, i.e., of high wavelength, would correspond a high value of the entropy. According to this supposition, under conditions in which the enthalpy of any transformation remains constant, there would be a spontaneous tendency of the entropy to grow, which means that a spectrum composed by radiations of high frequency would present a spontaneous tendency to convert itself in another of lower frequency radiations. The free energy term associated to this transformation (which can be identified with the amount of free energy dissipated through all the changes induced in the biosphere) can be represented by the product of the absolute temperature, $T$, multiplied by the entropy change occurring in it, $\Delta S$ (T $\Delta S=h \Delta v$, where $\Delta v$ denotes the change in frequency associated with any process induced by the changes in the characteristics of the radiation).

The thermodynamic formulation of the energy change which gives rise to every phenomenon occurring in the biosphere can be expressed according to the following equation [2]:

$$
\Delta G=\Delta H-T \Delta S=h \Delta v=\Delta E
$$

where $\Delta E$ represents the free energy dissipated as consequence of all the transformations which take place in the biosphere.

In the case of an infinitesimal change of the magnitudes produced under conditions of constant enthalpy ( $\mathrm{d} H=$ 0 ), the above equation reduces to the following expression:

$$
\mathrm{d} G=-T \mathrm{~d} S=h \mathrm{~d} v=\mathrm{d} E
$$

The result of the differentiation of the last equation with respect to the time (at constant temperature) gives rise to the rate of change of the free energy with the time (or the rate of free energy dissipation), which is given by the following expression. 


$$
\frac{\mathrm{d} G}{\mathrm{~d} t}=-T \frac{\mathrm{d} S}{\mathrm{~d} t}=h \frac{\mathrm{d} v}{\mathrm{~d} t}=\frac{\mathrm{d} E}{\mathrm{~d} t}
$$

Remembering that $c=\lambda v, v=c / \lambda, y: \mathrm{d} v=\left(-c / \lambda^{2}\right) \times \mathrm{d} \lambda$. By substitution of this equivalence in Equation (3) one obtains the following expression:

$$
\frac{\mathrm{d} G}{\mathrm{~d} t}=-T \frac{\mathrm{d} S}{\mathrm{~d} t}=h \frac{\mathrm{d} v}{\mathrm{~d} t}=-\frac{h c}{\lambda^{2}} \frac{\mathrm{d} \lambda}{\mathrm{d} t} .
$$

Finally, the rate of generation of entropy, measured by $(\mathrm{d} S / \mathrm{d} t)$ would be given by the following expression:

$$
\frac{\mathrm{d} S}{\mathrm{~d} t}=-\frac{1}{T} \frac{\mathrm{d} G}{\mathrm{~d} t}=\left(\frac{h c}{T \lambda^{2}}\right)\left(\frac{\mathrm{d} \lambda}{\mathrm{d} t}\right)=\left(\frac{h v}{T}\right)\left(\frac{\mathrm{d} \lambda}{\mathrm{d} t}\right)=(P C) \times\left(\frac{\mathrm{d} \lambda}{\mathrm{d} t}\right) .
$$

According to Equation (5) the rate of generation of entropy is proportional to the rate of change of the wavelength with the time. The coefficient of proportionality (denoted as $P C$ ) is always positive and is given by the following expression:

$$
(P C)=\left(\frac{h c}{T \lambda^{2}}\right)=\left(\frac{h v}{T \lambda}\right)
$$

This coefficient is an inverse function of the absolute temperature and would be measured in following units in the SI:

$$
\begin{aligned}
\left(\frac{h c}{T \lambda^{2}}\right) & =\frac{(\mathrm{J} \cdot \mathrm{s}) \times\left(\mathrm{kg} \cdot \mathrm{m}^{2} \cdot \mathrm{s}^{-2} \times \mathrm{J}^{-1}\right) \times\left(\mathrm{m} \cdot \mathrm{s}^{-1}\right)}{\mathrm{K} \cdot \mathrm{m}^{2}} \\
& =\mathrm{J} \cdot \mathrm{K}^{-1} \cdot \mathrm{m}^{-1} \cdot \mathrm{s}^{-2}=\left(\mathrm{kg} \cdot \mathrm{m} \cdot \mathrm{s}^{-2}\right) \cdot \mathrm{K}^{-1}=\mathrm{N} \cdot \mathrm{K}^{-1}
\end{aligned} .
$$

The fact that $(P C)>0$ implies that a growth of the entropy with the time, which is indicated by $(\mathrm{d} S / \mathrm{d} t)>0$ and expresses the spontaneous evolution of the entropy with the time in any system considered, would be the result of a growth of the wavelength with the time, i.e., would be the consequence of the positive value of the rate of change of the wavelength with the time, $(\mathrm{d} \lambda / \mathrm{d} t)>0$. In other words, it can be said that the higher the wavelength of a radiation the higher would be its content in entropy.

On the other hand, the proportionality coefficient in Equation (6) $\left[(P C)=\left(h c / T \lambda^{2}\right)=(h v / T \lambda)\right]$ is an inverse function of the square of the wavelength and of the temperature. Thus, the most energetic radiations component of the solar light (i.e., those radiations of higher frequency or of lower wavelength), would be those that would give rise to a more rapid free energy dissipation or a higher rate of generation of entropy. Likewise, according to Equation (5) the rate of generation of entropy grows proportionally to the inverse of the temperature.

Finding $\mathrm{d} S$ from Equation (5) one comes to following expression:

$$
\mathrm{d} S=\left(\frac{h c}{T \lambda^{2}}\right) \mathrm{d} \lambda=\left(\frac{h v}{T \lambda}\right) \mathrm{d} \lambda
$$

By integrating this equation it is possible to calculate the change of entropy $\left(\Delta S=S_{f}-S_{i n}\right)$, associated with a wavelength variation from an initial value of $\lambda=\lambda_{\text {in }}$ to a final value $\lambda_{f}$ :

$$
\begin{gathered}
\Delta S=S_{f}-S_{i n}=\int_{S_{i n}}^{S_{f}} \mathrm{~d} S=\left(\frac{h c}{T}\right) \int_{\lambda_{i n}}^{\lambda_{f}} \frac{\mathrm{d} \lambda}{\lambda^{2}}=\frac{h c}{T}\left(\frac{1}{\lambda_{\text {in }}}-\frac{1}{\lambda_{f}}\right) \\
=\Delta S=S_{f}-S_{i n}=\frac{h}{T}\left(v_{i n}-v_{f}\right) .
\end{gathered}
$$

According to this expression $\Delta S>0$ whenever $\lambda_{\text {in }}<\lambda_{f}$ or, in the case that the entropy change would be expressed as a function of the frequencies, whenever $v_{f}<v_{i n}$.

These expressions allow to perform calculations about the entropy changes which accompany to the conversion of an Avogadro number of photons of visible radiation of wavelength corresponding to the blue ( $\lambda_{\text {blue }}=430$ $\mathrm{nm}$ ) to an Avogadro number of photons of another radiation corresponding to the maximum in the far infrared spectrum corresponding to a black body at $15^{\circ} \mathrm{C}=288 \mathrm{~K}$, which is situated at about $\lambda_{\text {máx }}=20000 \mathrm{~nm}$. 


\section{Conclusions}

According to the ideas above developed, it could be said that the "arrow of the time" defined by Clausius [3] could be interpreted as the irremediable spontaneous conversion along the time of radiations of high frequency, i.e., of shorter wavelengths, in radiations of lower frequencies, i.e., of longer wavelengths, which are those emitted by a black body at $15^{\circ} \mathrm{C}$, as the biosphere can be supposed to be. Therefore, the energy necessary to maintain all the processes taking part in the biosphere should be attributed to the free energy changes proceeding from the term $\mathrm{T} \Delta \mathrm{S}$, i.e., from a change in the radiation spectrum corresponding to the sun surface (around 6000 $\mathrm{K})$ to the radiation spectrum of a black body at $288 \mathrm{~K}$. In this case, in which it can be supposed that the enthalpy variation is equal to null, the driving force for the spontaneous transformation of short-length-radiations in radiations of longer wavelengths is the increase of entropy associated with such a change.

It can be said that the "quality" or grade of the energy transported by the electromagnetic radiation is inversely proportional to the wavelength, or, directly proportional to the frequency of the radiation considered. According to the second principle of thermodynamics, there is a natural tendency to the energy to spontaneously degrade and to convert itself from a higher into a lower grade or "noblesse" [4]. This degradation in the "noblesse" of the energy explains the origin of the work amount necessary for driving the multiple processes and phenomena taking place in the biosphere, whereas no thermal energy change can be observed.

According to the arguments above exposed, also the so called cosmic microwave radiation background that according to S. Weinberg [5] could be considered as a black-body radiation with a temperature of $3 \mathrm{~K}$, which could be the consequence of the spontaneous degradation of electromagnetic radiations of higher frequencies produced along the time.

\section{References}

[1] Twidell, J. and Weir, T. (2006) Renewable Energy Resources. 2nd Edition, Taylor and Francis, London, New York.

[2] Sears, F.W. (1952) An Introduction to Thermodynamics, the Kinetic Theory of Gases, and Statistical Mechanics. Addison Wesley Publishing Company, Massachusetts.

[3] Castellan, G.W. (1971) Physical Chemistry. Addison Wesley Publishing Company, Reading.

[4] González Velasco, J. (2009) Energías Renovables. Ed. Reverté, Barcelona.

[5] Weinberg, S. (1980) The First Three Minutes. A Modern View of the Origin of the Universe. Bentam Books, New York. 